verfassungsrechtlich nicht nur zulässig, sondern auch geboten, weil die Grundrechte nicht ausschließlich abwehrrechtlich zu interpretieren sind, sondern auch staatliche Schutzpflichten erzeugen. Bislang wurden zum Schutz der Medienfreiheiten des Art. 5 Abs. 1 Satz 2 GG geeignete Maßnahmen nicht ergriffen, so dass das Untermaßverbot verletzt ist. Da Hauptzweck der angeregten gesetzlichen Neuregelung die Beschränkung der allgemeinen Parteienfreiheit ist, ist eine Änderung des Parteiengesetzes erforderlich.

\title{
Das rechtliche Ende politischer Parteien: Auflösung und Verschmelzung
}

\author{
Martin Limpert ${ }^{*}$
}

Das Parteiengesetz kennt vier Formen des Erlöschens von politischen Parteien: die Auflösung als Selbstauflösung, als Zwangsauflösung in einem Parteiverbotsverfahren und als Auflösung kraft Gesetzes sowie die Verschmelzung. Für die Konstellationen der Selbstauflösung, der Zwangsauflösung und der Verschmelzung gibt es in jüngster Zeit konkrete Fälle im Parteiensystem der Bundesrepublik Deutschland. Die Partei Die Grauen - Graue Panther (Kurzbezeichnung: Graue) hat sich aufgelöst. Die Linke ist durch Verschmelzung der Parteien Die Linkspartei.PDS und Wahlalternative Arbeit und soziale Gerechtigkeit (WASG) entstanden. Gegen die Nationaldemokratische Partei Deutschlands (NPD) ist ein Verbotsverfahren vor dem Bundesverfassungsgericht (BVerfG) eingeleitet worden, das wegen eines nicht behebbaren Verfahrenshindernisses eingestellt wurde.

\section{Auflösung}

\subsection{Selbstauflösung der Partei Graue}

Die Grauen befinden sich derzeit vermögensrechtlich in Liquidation. Ein Delegiertenparteitag beschloss am 1. März 2008 mit Mehrheit (42 von 57 der Stimmberechtigten) die Auflösung der Partei. ${ }^{1}$ Die am 25. März 2008 vorgenommene Auszählung der Urabstimmung ergab, dass von den 417 teilnehmenden Parteimitgliedern 342 für die Selbstauflösung gestimmt hatten. ${ }^{2}$ Die nach $\$ 17$ Abs. 1 der Satzung der Partei notwendige Zweidrittelmehrheit war damit erreicht. Am 29. März 2008 ist ein Auflösungsprotokoll verfasst und dem Bundeswahlleiter zugeleitet worden. Bereits am 25. März hatte der Bundesvorstand der Grauen ihren bisherigen ersten stellvertretenden Bundesvorsitzenden zum Liquidator bestellt.

Am 1. März 2008 ist in Berlin eine Partei unter dem Namen „Die Grauen - Generationspartei“ gegründet worden, die sich als Nachfolgerin der Partei Graue versteht, wie

\footnotetext{
Der Autor, Angehöriger der Verwaltung des Deutschen Bundestags, gibt in dem Beitrag ausschließlich seine persönliche Ansicht wieder.

1 Vgl. Meldung des ddp vom 2. März 2008.

2 Vgl. Meldung der dpa vom 25. März 2008.
} 
deren ehemaliger Bundesvorsitzende mitteilte. Am 29. März 2008 haben 34 ehemalige Mitglieder der Grauen in Fulda die Partei „Allianz Graue Panther“ ins Leben gerufen. ${ }^{3}$

\subsection{Verfahren der Auflösung}

Das Parteiengesetz (PartG) selbst regelt das Verfahren der Auflösung einer Partei nur rudimentär. Es verweist die damit zusammenhängenden Regelungen weitgehend in den Bereich der Satzungsautonomie der Partei, indem es in $₫ 6$ Abs. 2 Nr. 11 PartG bestimmt, dass die Satzungen Bestimmungen enthalten müssen über eine Urabstimmung der Mitglieder und das Verfahren, wenn der Parteitag die Auflösung der Partei oder des Gebietsverbandes oder die Verschmelzung mit anderen Parteien nach $\$ 9$ Abs. 3 PartG beschlossen hat.

Immerhin stellt $\$ 6$ Abs. 2 Nr. 11 PartG als Spezialbestimmung für den Umgang mit der Existenzfrage einer Partei damit zwei Anforderungen an das Verfahren: Es muss zunächst ein Auflösungsbeschluss des Parteitages nach $\$ 9$ Abs. 3 PartG gefasst werden und dann eine Urabstimmung der Mitglieder erfolgen. Die Einzelheiten sind in der Satzung zu bestimmen, so etwa das notwendige Quorum in der Urabstimmung, um einen Auflösungsbeschluss des Parteitags für bestätigt zu erachten. Wird das in der Satzung festgelegte Mehrheitserfordernis nicht erreicht, ist die vom Parteitag beschlossene Auflösung oder die Fusion gescheitert. ${ }^{4}$

Der Parteitagsbeschluss gilt gemäß $₫ 6$ Abs. 2 Nr. 11 S. 2 PartG nach dem Ergebnis der Urabstimmung als bestätigt, geändert oder aufgehoben. Das Datum der Auflösung ist nicht der Zeitpunkt der Urabstimmung, sondern der des Parteitagsbeschlusses oder das Datum, das in dem Parteitagsbeschluss als Auflösungsdatum festgelegt ist ${ }^{5}$, im Falle der Partei Graue der 29. März 2008.

Die Satzung der Grauen (Stand: 23. Januar 2008) bestimmte in $\$$ 17.1.: „Hat der Parteitag die Auflösung der Partei oder die Verschmelzung mit einer oder mehreren anderen Parteien beschlossen, bedarf dies der Bestätigung durch eine schriftliche Urabstimmung aller Mitglieder, die zum Abstimmungszeitpunkt ihr aktives Wahlrecht besitzen. Der Abstimmungszeitpunkt wird vom Bundesvorstand festgelegt. Die Urabstimmung ist spätestens drei Monate nach dem Parteitagsbeschluss durchzuführen. Die Bestätigung des Parteitagsbeschlusses bedarf einer Mehrheit von zwei Dritteln der schriftlich abgegebenen Stimmen."

Im Falle der Auflösung der Partei teilen ihre Untergliederungen das Schicksal der Gesamtpartei. $\$ 7$ Abs. 1 PartG beinhaltet ein objektives, an die Parteien gerichtetes Organisationsgebot, wonach sie sich in Gebietsverbände zu gliedern haben. Größe und Umfang der Gebietsverbände werden durch die Satzung festgelegt. Die gebietliche Gliederung muss so weit ausgebaut sein, dass den einzelnen Mitgliedern eine angemessene Mitwirkung an der Willensbildung der Partei möglich ist; sie muss jedenfalls bis zur Kreisstufe reichen. ${ }^{6}$

3 Vgl. Meldung der dpa vom 29. März 2008.

4 Vgl. Martin Morlok, Kommentar zum Gesetz über die politischen Parteien, in: Josef Kölble (Hrsg.), Das Deutsche Bundesrecht, Baden-Baden 2007, Erläuterungen zu $₫ 6$ PartG Rn. 11.

5 Vgl. ebenda.

6 Vgl. Karl-Heinz Seifert, Die politischen Parteien im Recht der Bundesrepublik Deutschland, Köln 1975, S. 201. 
Da die Parteimitgliedschaft nur natürlichen Personen gewährt werden kann, können Gebietsverbände nicht Mitglieder der Gesamtpartei, sondern nur deren Gliederungen beziehungsweise Teilorganisationen der Gesamtpartei sein. ${ }^{7}$

Demzufolge gliederte sich die Partei Graue in den Bundesverband sowie in Landes-, Kreis- und Ortsverbände ( $\$$ 9.1. der Satzung). Dabei umfasste der Bundesverband das gesamte Gebiet der Bundesrepublik Deutschland. Die Landesverbände entsprachen den Gebieten der Bundesländer ( $\$$ 9.2. der Satzung). Die Gliederungen waren rechtlich unselbstständige Teile der Bundespartei und besaßen keine eigenen Rechte und Pflichten, soweit das Parteiengesetz und die geltenden gesetzlichen Bestimmungen keine zwingenden, hiervon abweichenden Anordnungen treffen ( $\$$ 9.1. der Satzung). Derartige zwingende gesetzliche Regelungen, die etwas Abweichendes gebieten, sind nicht ersichtlich.

Als so genannter Gesamtverein umfasst die (Gesamt-)Partei die jeweils nachgeordneten Organisationsstufen in Form der gemäß $₫ 7$ Abs. 1 Satz 1 PartG zu bildenden Gebietsverbände. Mit dem Vereinsstatus verbindet der Gesetzgeber die Forderung nach einer verbandsmäßigen Untergliederung zur Verwirklichung der innerparteilichen Demokratie. Daraus folgt, dass den Untergliederungen ein Bereich selbstständiger Willensbildung und Selbstverwaltung gewährt werden muss. Eine uneingeschränkte Kompetenz würde indes zu Kollisionen mit dem Funktionserhaltungsinteresse der politischen Parteien und mit der Möglichkeit zu einem einheitlichen und geschlossenen Auftreten führen. ${ }^{8}$ Deshalb ist in nahezu allen Parteisatzungen die Bindung der Untergliederungen an Satzung, Programm oder Beschlüsse der höheren Organisationsstufen verankert und als selbstverständlich anzusehen. ${ }^{9}$ In der Satzung der Grauen heißt es dazu (\$ 9.4.): „Das Recht der Gliederung zur eigenverantwortlichen politischen Arbeit innerhalb der durch die Satzung, die Beschlüsse der Parteitage und das Parteiprogramm vorgegebenen Grenzen und die Haftung aller handelnden Personen, insbesondere im Falle einer Verletzung der von ihnen übernommenen Pflichten, bleibt hiervon unberührt."

Aus der hierarchischen Organisationsstruktur ergibt sich, dass der Beschluss zur Auflösung der Partei alle ihre Untergliederungen erfasst. Eine Bestandsgarantie zugunsten der einmal festgelegten Gliederung ist nicht anzunehmen. Dadurch würden nämlich Reaktionsmöglichkeiten auf eingetretene reale äußere wie innere Veränderungen unzulässig beschnitten. ${ }^{10}$ So haben ja auch die jeweils zuständigen Organisationsstufen die Möglichkeit, einzelne Gebietsverbände aufzulösen, soweit hierfür eine organisatorische Notwendigkeit besteht, etwa als Reaktion auf eine allgemeine (staatliche) Gebietsreform. ${ }^{11}$ Dies ergibt sich unter anderem aus $\$ 6$ Abs. 2 Nr. 11 PartG, wonach die Satzungen Bestimmungen über die Auflösung der Partei oder eines Gebietsverbandes enthalten müssen. Nach $\$ 6$ Abs. 3 Nr. 3 PartG ist der Vorstand verpflichtet, dem Bundeswahlleiter die Auflösung der Partei oder eines Landesverbandes mitzuteilen. Umgekehrt haben die Parteimitglieder gegenüber

7 Vgl. ebenda, S. 205; Bernhard Reichert, Handbuch Vereins- und Verbandsrecht, Neuwied 2007, Rn. 5638 f.

8 Vgl. Martin Morlok, a.a.O. (Fn. 4), Erläuterungen zu \$7 PartG Rn. 4.

9 So Karl-Heinz Seifert, a.a.O. (Fn. 6), S. 273.

10 Vgl. Martin Morlok, a.a.O. (Fn. 4), Erläuterungen zu $\$ 7$ PartG Rn. 11.

11 Eine solche Maßnahme ist zu unterscheiden von der Auflösung nach $₫ 16$ Abs. 1 Satz 1 PartG, die als Disziplinarmaßnahme nur bei schwerwiegenden Verstößen gegen die Grundsätze oder die Ordnung der Partei zulässig ist. 
der Partei keinen Anspruch auf die Gründung eines bestimmten (zum Beispiel Gemeinde-) Verbandes. $\$ 7$ Abs. 1 Satz 3 PartG verlangt lediglich den Ausbau der gebietlichen Gliederung so weit, dass den einzelnen Mitgliedern eine angemessene Mitwirkung an der Willensbildung der Partei möglich ist. Ein subjektives Recht des einzelnen Mitglieds oder einer Gruppe von Mitgliedern wird dadurch nicht begründet. ${ }^{12}$

\subsection{Liquidation der Partei}

Mit ihrer Auflösung erlischt die Partei, und zwar die Gesamtpartei und ihre Gliederungen. Die Partei verliert damit den Status nach Art. 21 GG. Der Verein befindet sich in Liquidation. Diese erfolgt nach den Regeln des bürgerlichen Vereinsrechts, wie sich aus $\$ 37$ PartG ergibt. Nach dieser Vorschrift wird $\$ 54$ Satz 2 BGB, der die Handelndenhaftung beim nichtrechtsfähigen Verein regelt, nicht angewandt. Daraus ist im Umkehrschluss zu folgern, dass die übrigen Vorschriften des bürgerlichen Vereinsrechts auf Parteien Anwendung finden. Ein weiterer Verweis auf das BGB findet sich in $\$ 11$ Abs. 3 PartG, der die Vertretung der Gebietsverbände betrifft und auf $\$ 26$ Abs. 2 BGB Bezug nimmt. Es gilt also das private Vereinsrecht, soweit nicht vorrangig Art. 21 GG und das Parteiengesetz etwas anderes bestimmen. ${ }^{13}$

Da $\$ 54$ Satz 2 BGB gemäß $\$ 37$ PartG für Parteien nicht gilt, ist die haftungsrechtliche Unterscheidung zwischen rechtsfähigem Verein und nichtrechtsfähigem Verein im Außenverhältnis aufgehoben. ${ }^{14}$ Der Geschädigte ist auf die Haftung des Vereins mit dem Vereinsvermögen beschränkt. ${ }^{15} \$ 54$ Satz 2 BGB bezieht sich allerdings nur auf die rechtsgeschäftliche Haftung. Im deliktischen Bereich wendet die inzwischen herrschende Meinung ${ }^{16}$ die Haftungszurechnungsnorm des $₫ 31$ BGB entsprechend an. Sie begründet lediglich eine Haftung des nichtrechtsfähigen Vereins, nicht aber der Mitglieder persönlich. Entscheidendes Kriterium für die Anwendung von $\$ 31$ BGB ist die eigenverantwortliche weisungsfreie Tätigkeit des Handelnden. ${ }^{17}$ Auch soweit der Verein aus $\$ 831$ BGB wegen des Verhaltens eines Verrichtungsgehilfen in Anspruch genommen wird, haftet ausschließlich das Sondervermögen. Beim nichtrechtsfähigen Idealverein ist die Haftung daher in der Regel auf das Vereinsvermögen beschränkt. ${ }^{18}$

Haftungsmasse der Partei Graue ist ihr gesamtes Vermögen. Dazu bestimmt $₫$ 18.1. Unterabsatz 1 der Satzung: „Die Partei ist Eigentümer aller vorhandenen Gelder und des sonstigen Vermögens. Zu Verfügungen über das Vermögen der Partei ist nur der Vorstand,

12 Karl-Heinz Seifert, a.a.O. (Fn. 6), S. 201; vgl. auch das Bundesparteigericht der CDU, BPG 4/86 vom 30. April 1987, in den Gründen zu II. 2.

13 Allgemeine Meinung; vgl. Wilhelm Henke, in: Rudolf Dolzer / Hans Jürgen Abraham (Hrsg.), Kommentar zum Bonner Grundgesetz, Bd. 5, Heidelberg 1991, Art. 21 Rn. 219; Bernhard Reichert, a.a.O. (Fn. 7), Rn. 5829; Martin Morlok, a.a.O. (Fn. 4), Erläuterungen zu $\$ 37$ PartG Rn. 1.

14 Vgl. Dieter Reuter, in: Franz Jürgen Säcker / Roland Rixecker (Hrsg.), Münchener Kommentar zum Bürgerlichen Gesetzbuch, Bd. 1, München 2006, \$54 Rn. 16 f.

15 Vgl. Martin Morlok, a.a.O. (Fn. 4), Erläuterungen zu $₫ 37$ PartG Rn. 1.

16 Vgl. Otto Palandt / Helmut Heinrichs, Bürgerliches Gesetzbuch, München 2009, $\$ 54$ Rn. 12; Bernhard Reichert, a.a.O. (Fn. 7), Rn. 5831.

17 Vgl. Otto Palandt / Helmut Heinrichs, a.a.O. (Fn. 16), \$31 Rn. 6.

18 Vgl. Oberlandesgericht Hamm, Wertpapiermitteilungen 85, S. 644. 
vertreten durch das Präsidium, berechtigt. Er ist berechtigt, in eigenem Namen und aus eigenem Recht alle der Partei zustehenden Ansprüche gegen Schuldner der Partei geltend zu machen.“ In Unterabsatz 2 heißt es: „Über beziehungsweise für die Gelder, die den Landesverbänden aus der staatlichen Parteienfinanzierung zufließen, verfügen und haften das jeweilige Landespräsidium und der jeweilige Landesvorstand.“

Danach ist Inhaberin des Vermögens die Gesamtpartei. Verfügungsberechtigt ist der Bundesvorstand, vertreten durch das Präsidium. Die Landesverbände haben kein eigenes Vermögen. Das jeweilige Landespräsidium und der jeweilige Landesvorstand sind aber über den Teil des Vermögens der Gesamtpartei verfügungsbefugt, der dem Landesverband durch die staatliche Parteienfinanzierung des betreffenden Landes zufließt.

Wenn kein Vermögen an den Fiskus fällt, erfolgt die Liquidation eines nichtrechtsfähigen Vereins entsprechend $\$ \$ 47$ ff. BGB, sofern nicht die Satzung ausdrücklich die Vermögensauseinandersetzung nach dem Recht der Personengesellschaft des bürgerlichen Rechts vorsieht. ${ }^{19}$ Eine entsprechende Bestimmung enthält die Satzung der Grauen nicht. Die Liquidatoren haben alle bei Eintritt des Beendigungsgrundes vorhandenen und während der Abwicklung entstandenen rechtsgeschäftlichen und gesetzlichen Verbindlichkeiten des Vereins zu erfüllen ( $\$ \$ 362$ ff. BGB). Erweist sich, dass das Vereinsvermögen zur Befriedigung sämtlicher Gläubiger nicht ausreicht, haben sie gemäß $\$ \$ 42$ Abs. 2, 48 Abs. 2 BGB Insolvenzantrag zu stellen. ${ }^{20}$

\subsection{Auflösung und anschließende Neugründung}

Das Selbstauflösungsrecht rechtsfähiger Vereine ist unabdingbar ( $\$ 41 \mathrm{BGB})$; für nichtrechtsfähige Vereine ist das Selbstauflösungsrecht nicht zwingend. ${ }^{21}$ Die Satzung einer Partei kann deshalb für ihre Untergliederungen, die nichtrechtsfähige Vereine sind, bindend und für diese unabänderlich vorschreiben, dass entweder nur die Gesamtpartei oder die „höhere“ Untergliederung die „niedrigere“ aufösen kann. Der Gesamtpartei als nicht rechtsfähigem Verein kann aber das Selbstauflösungsrecht nicht vorenthalten werden, wie sich auch aus dem Parteiengesetz ergibt, das die Selbstauflösung an mehreren Stellen erwähnt ( $\$ \$ 6$ Abs. 2 Nr. 11, Abs. 3 Nr. 3 und 9 Abs. 3 PartG). Diese Bestimmungen normieren materiellrechtlich keine Gründe, deren Vorliegen Voraussetzung für die Auflösung der Partei wäre. Die Wahrnehmung des Selbstauflösungsrechts einer Partei ist auch verfassungsrechtlich an keine Gründe gebunden. Es handelt sich gewissermaßen um ein „Grundrecht" der Partei aus Art. 21 GG, das der Gründungsfreiheit korrespondiert. ${ }^{22}$ Das BVerfG führte dazu aus: „Die Parteienfreiheit umfasst die freie Wahl der Rechtsform, der inneren Organisation sowie der Zielsetzung einschließlich Name, Satzung und Programm, die Teilnahme an Wahlen sowie die Verfügung über Einnahmen und Vermögen. In personeller

19 Vgl. BGHZ 50, S. 325 (S. 329); Bernhard Reichert, a.a.O. (Fn. 7), Rn. 4836.

20 Vgl. Hans Theodor Soergel / Walther Hadding, Bürgerliches Gesetzbuch, Stuttgart 2000, $\$ 49$ Rn. 7.

21 Oberlandesgericht München, in: DÖV 1972, S. 361.

22 Hans Hugo Klein, in: Theodor Maunz / Günter Dürig, Grundgesetz. Kommentar, München 2001, Art. 21 Rn. 273; Rudolf Streinz, in: Hermann von Mangoldt / Friedrich Klein / Christian Starck, Das Bonner Grundgesetz. Kommentar, Bd. 2, München 2003, Art. 21 Rn. 103. 
Hinsicht verbürgt sie die freie Entscheidung über Aufnahme und Ausschluss von Mitgliedern bis hin zur Selbstauflösung der Partei und der Vereinigung mit anderen Parteien. "23 Ebenso wie ihre Selbstaufösung im freien Ermessen einer Partei liegt, kann sie sich anschließend wieder neu gründen. Der damit verfolgte Zweck unterliegt keiner vorangehenden staatlichen Prüfung. Selbst die Gründung einer Partei, die verfassungsfeindliche Ziele verfolgt, ist frei, wenngleich verfassungswidrig. ${ }^{24}$ Da über die Frage der Verfassungswidrigkeit aber allein das BVerfG in einem Verbotsverfahren entscheidet, kann dagegen nur repressiv und nicht etwa präventiv in einem Prüfverfahren vorgegangen werden. ${ }^{25}$

Die Gründungsfreiheit des Art. 21 Abs. 1 Satz 2 GG ist an keine ausdrücklichen Schranken gebunden. Solche ergeben sich aus Art. 21 Abs. 1 Satz 3 GG (demokratische Grundsätze) für die innere Organisation (vgl. die Konkretisierung in $\$ 6$ ff. PartG) und aus dem verfassungsrechtlichen Parteibegriff ${ }^{26}$ für die Wahl der Rechtsform, die eine körperschaftliche Struktur unter Ausschluss öffentlich-rechtlicher Rechtsformen, somit einen der Organisationstypen des bürgerlichen Vereinsrechts erfordert. Diese Einschränkungen sichern die Verfassungsfunktion der Parteien und sind ebenso zulässig wie die grundsätzliche Geltung zivilrechtlicher Ordnungsbestimmungen. ${ }^{27}$ Letztere werden insoweit modifiziert, als es die Besonderheiten politischer Parteien gebieten. ${ }^{28}$ Die Anwendung der ordnungsrechtlichen Vorschriften zwecks Gründung stehen (auch) wegen Art. 21 Abs.1 Satz 2 GG unter einem Missbrauchsvorbehalt, denn das Verfassungsrecht „will“ die Parteiengründung. ${ }^{29}$ Als zulässig erachtet werden insbesondere die Anforderungen an Satzung und Programm der Partei nach $\$ 6$ PartG sowie an ihren Namen nach $\$ 4$ PartG. Da die Auswahl an aussagekräftigen Parteinamen begrenzt ist, dürfen die Anforderungen an die von $\$ 4$ Abs.1 PartG geforderte Unterscheidbarkeit gegenüber Namen und Kurzbezeichnung einer bereits bestehenden Partei nicht überzogen werden. ${ }^{30}$ Für die Frage, ob eine Verwechslungsgefahr besteht, kommt es auf den Gesamteindruck an, den die beiden nach Klang und Sinngehalt auf den Verkehr hervorrufen. ${ }^{31}$ Zulässig sind auch die formellen Anforderungen an die Auflösung der Partei. ${ }^{32}$

Nach alldem kann die Gründung einer Nachfolgepartei der Grauen rechtlich nicht beanstandet werden. Die Verbindlichkeiten der Partei Graue gehen nicht auf die Nachfolgepartei über.

23 BVerfG, Beschluss vom 28. März 2002, in: NJW 2002, S. 2227.

24 Vgl. Hans Hugo Klein, a.a.O. (Fn. 22), Art. 21 Rn. 273.

25 Vgl. Rudolf Streinz, a.a.O. (Fn. 22), Art. 21 Rn. 104.

26 Vgl. statt aller ebenda, Art. 21 Rn. 53 f.

27 Vgl. ebenda, Art. 21 Rn. 104; zum Verhältnis des Parteienrechts zum Zivilrecht vgl. Wilhelm Henke, a.a.O. (Fn. 13), Art. 21 Rn. 219.

$28 \$ 37$ PartG erklärt $\$ 54$ Satz 2 BGB (persönliche Haftung des im Namen des Vereins Handelnden) für nicht anwendbar. Die in $\$ 37$ alter Fassung ebenfalls genannten $\$ \$ 61-63$ BGB (Einspruchsrecht der Verwaltungsbehörde bei Eintragungen) wurden durch Art. 11 JuMiG vom 18. Juni 1997 (BGBl. I, S. 1430) aufgehoben.

29 Philip Kunig, in: Ingo von Münch / ders., Grundgesetz-Kommentar, Bd. 2, München 2001, Art. 21 Rn. 47.

30 Vgl. Karl-Heinz Seifert, a.a.O. (Fn. 6), S. 273.

31 Vgl. Landgericht Bremen, in: NJW 1989, S. 1864 (S. 1865).

32 Vgl. Rudolf Streinz, a.a.O. (Fn. 22), Art. 21 Rn. 104. 


\subsection{Parteispaltung}

Als Unterfall der Selbstauflösung einer Partei kann die Parteispaltung (Dismembration) angesehen werden. Sie ist zu unterscheiden von der Parteiabspaltung (Sezession). Die Abspaltung von Gruppen steht zwischen der Parteispaltung und dem Ausscheiden einzelner Mitglieder aus der Partei durch Ausschluss oder Austritt.

Weder das Bundeswahlgesetz (BWG) noch das Parteiengesetz kennen den Begriff Parteispaltung. Sie ist gesetzlich nicht bedacht worden ${ }^{33}$, obwohl sie in der politischen Wirklichkeit der fünfziger Jahre eine weit größere Rolle gespielt hat als einzelne Parteiaustritte oder Parteiausschlüsse. ${ }^{34}$ Es handelt sich also nicht um eine rein akademische Unterscheidung. Sie wird vor allem im Wahlrecht bei der Nachrückerproblematik virulent. Nach $\$ 48$ Abs. 1 Satz 2 BWG bleiben bei der Nachfolge eines ausgeschiedenen Abgeordneten diejenigen Listenbewerber unberücksichtigt, die seit dem Zeitpunkt der Aufstellung der Landeslisten aus der betreffenden Partei ausgeschieden sind. Das „Ausscheiden“ im Sinne dieser Parteiklausel bildet demnach den Oberbegriff für alle Modalitäten eines Verlustes der Mitgliedschaft in einer Partei. ${ }^{35}$

In der Diskussion um den (juristischen, nicht soziologischen) Begriff und die Bedeutung einer Parteispaltung für die Listennachfolge ist unumstritten, dass $\$ 48$ Abs. 1 Satz 2 BWG keine direkte Anwendung findet. ${ }^{36}$ Unterschiedlich beurteilt wird die Frage einer analogen Anwendung der Vorschrift. Gelegentlich wird die Analogie damit begründet, dass Parteispaltung und -neugründung „wegen des Momentes der selbst bestimmten Veränderungen der Ausgangslage wie ein freiwilliger Austritt zu behandeln“ seien. ${ }^{37}$ Die wohl überwiegende Auffassung geht aber dahin, dass $\$ 48$ Abs. 1 Satz 2 BWG auf den Fall der Parteispaltung nicht zugeschnitten sei. ${ }^{38}$ Abspaltungen werden als Gruppenaustritte beziehungsweise Gruppenausschlüsse und damit als bloße Häufung von freiwilligen Austritten beziehungsweise unfreiwilligen Ausschlüssen einzelner Parteimitglieder in direkter Anwendung des $\$ 48$ Abs. 1 Satz 2 BWG gewertet. Insbesondere werden gegen eine Anwendung der Parteiklausel auf die Parteispaltung „beweistechnische Schwierigkeiten“ angeführt, da nicht feststellbar sei, ob die neue Gruppierung oder die in der alten Partei verbliebenen Mitglieder das ursprüngliche Programm fortführten. ${ }^{39}$ Zudem würde die Anwendung der Parteiklausel die „oft notwen-

33 Vgl. Martin Morlok, in: Horst Dreier, Grundgesetz. Kommentar, Bd. II, Tübingen 2006, Art. 38, Rn. 78.

34 Vgl. Otto Uhlitz, Über die Unvereinbarkeit des $\$ 48$ Abs. 1 Satz 2 des Bundeswahlgesetzes mit dem Grundgesetz, in: DÖV 1957, S. 468 (S. 469).

35 Wolfgang Schreiber, $\$ 48$ Abs. 1 Satz 2 Bundeswahlgesetz verfassungswidrig?, in: DVBl. 1976, S. 734 (S. 736); Peter Unruh, Partei und Mandat: Probleme der Listennachfolge, in: ZParl, 34. Jg. (2003), H. 1, S. $166-186$, S. 167.

36 Vgl. Peter Unruh, a.a.O. (Fn. 35), S. 168.

37 Martin Morlok, a.a.O. (Fn. 33), Art. 38, Rn. 78. Ähnlich geht das HbgVerfG (Urteil vom 2. Juli 2001, NVwZ-RR 2001, S. 697 (S. 698) vor, indem es allein auf den freiwilligen Austritt des Beschwerdeführers abstellt und das Vorbringen, es handle sich vorliegend um eine Parteispaltung, für irrelevant erklärt.

38 Vgl. Jens Schuster, Die Rechtsstellung der Ersatzleute zum Deutschen Bundestag, Erlangen 1967, S. 115; Otto Uhlitz, a.a.O. (Fn. 34), S. 469 f.; Wolfgang Schreiber, Handbuch des Wahlrechts zum Deutschen Bundestag, $\$ 48$ Rn. 5; Peter Unruh, a.a.O. (Fn. 35), S. 168 f.

39 Jens Schuster, a.a.O. (Fn. 38), S. 116; Peter Unruh, a.a.O. (Fn. 35), S. 171 f. 
digen Fortbildungen und politischen Flurbereinigungen " 40 verhindern. Im Übrigen liegen schon die Voraussetzungen einer Analogie nicht vor, will man darunter die „Anwendung einer Rechtsnorm mit anderen Tatbestandsvoraussetzungen auf einen ähnlichen, ungeregelten Sachverhalt“ ${ }^{41}$ verstehen. „Die Übertragung gründet sich darauf, dass infolge ihrer Ähnlichkeit in den für die gesetzliche Bewertung maßgeblichen Hinsichten beide Tatbestände gleich zu bewerten sind, also auf die Forderung der Gerechtigkeit, Gleichartiges gleich zu behandeln. " 42 Bei der Parteispaltung und dem Ausscheiden aus einer Partei im Sinne des $\$ 48$ Abs. 1 Satz 2 BWG handelt es sich nicht um Tatbestände, die in diesem Sinne vergleichbar wären, denn die Parteispaltung ist gegenüber dem von der Parteiklausel umfassten Sachverhalt des Verlustes der Mitgliedschaft in einer Partei ein Aliud. ${ }^{43}$

$\mathrm{Zu}$ diesem Ergebnis gelangt man, wenn man zur Gewinnung eines handhabbaren und justiziablen Begriffs der Parteispaltung ausschließlich auf formelle beziehungsweise äußerlich evidente Momente abstellt. ${ }^{44}$ Danach liegt eine Parteispaltung nur dann vor, wenn die Ursprungspartei formal beziehungsweise faktisch aufgelöst wird und aus ihren Teilen neue Gruppierungen mit Parteistatus entstehen, die sich der ursprünglichen Partei nicht mehr zuordnen lassen. Im Falle einer bloßen Abspaltung existiert die Ursprungspartei nach dem Ausscheiden einer Vielzahl ihrer Mitglieder formal/faktisch weiter. Die Wahlprüfungsorgane sind bei dieser formellen Betrachtungsweise der Entscheidung enthoben, wer noch personell oder ideologisch - die ursprüngliche (Listen-)Partei repräsentiert. Das Gebot der Klarheit und Eindeutigkeit des Wahlrechts wird so gewahrt, wenn das Fortbestehen der Ursprungspartei zweifelsfrei ausgeschlossen werden kann. Als Indizien bieten sich dafür Veränderungen im Parteienregister, der Organisationsstruktur oder des Namens der betreffenden Partei an.

\subsection{Zwangsauflösung}

Anders als im Falle der Selbstauflösung ist die Rechtslage bei vom Bundesverfassungsgericht verbotenen Parteien. Nach $₫ 33$ Abs. 1 PartG ist es verboten, Organisationen zu bilden, die verfassungswidrige Bestrebungen einer nach Art. 21 Abs. 2 GG in Verbindung mit $\$ 46$ BVerfGG verbotenen Partei an deren Stelle weiter verfolgen (Ersatzorganisationen), oder bestehende Organisationen als Ersatzorganisationen fortzuführen.

Erweist sich der Verbotsantrag als begründet, so stellt das BVerfG gemäß $₫ 46$ Abs. 1 BVerfGG fest, dass die Partei verfassungswidrig ist. Nach $\$ 46$ Abs. 2 BVerfGG kann die Feststellung auf einen rechtlich oder organisatorisch selbstständigen Teil einer Partei beschränkt werden. Mit der Feststellung ist gemäß $\$ 46$ Abs. 3 Satz 1 BVerfGG die Auflösung der Partei oder des selbstständigen Teils der Partei zu verbinden sowie das Verbot, eine Ersatzorganisation zu schaffen. Außerdem kann das BVerfG die Einziehung des Vermögens

40 So Jens Schuster, a.a.O. (Fn. 38), S. 116.

41 Bernd Rüthers, Rechtstheorie. Begriff, Geltung und Anwendung des Rechts, München 2008, Rn. 889.

42 Karl Larenz, Methodenlehre der Rechtswissenschaft, Berlin u.a. 1992, S. 269.

43 Vgl. Otto Uhlitz, a.a.O. (Fn. 34), S. 470; Peter Unruh, a.a.O. (Fn. 35), S. 172.

44 So zutreffend Peter Unruh, a.a.O. (Fn. 35), S. 170; Stellungnahme der Hamburger Behörde für Inneres in Drs. 16/4827 vom 31. Oktober 2000, S. 3. 
der Partei oder ihres selbstständigen Teils zugunsten des Bundes oder des Landes zu gemeinnützigen Zwecken gemäß $\$ 46$ Abs. 3 Satz 2 BVerfGG aussprechen.

Das Parteiverbot besteht also aus drei beziehungsweise vier Elementen, die im Urteilstenor gesondert auszusprechen sind: die Feststellung der Verfassungswidrigkeit, die Auflösung, das Verbot der Schaffung einer Ersatzorganisation und gegebenenfalls die Einziehung des Parteivermögens.

Das Verbotsverfahren als solches ist zweistufig: (1) \$45 BVerfGG sieht eine gerichtliche Vorprüfung vor, in der das BVerfG dem Vertretungsberechtigten der Partei - nicht der Partei selbst - Gelegenheit zur Äußerung binnen einer zu bestimmenden Frist gibt und dann beschließt, ob der Antrag als unzulässig oder als nicht hinreichend begründet zurückzuweisen oder ob die Verhandlung durchzuführen ist. Für den Beschluss, die Verhandlung durchzuführen, ist eine Zweidrittelmehrheit gemäß $\$ 15$ Abs. 4 Satz 1 BVerfGG erforderlich, da es sich um eine für den Antragsgegner nachteilige Entscheidung handelt. Es ist die gesetzliche Mitgliederzahl des zuständigen Senats zugrunde zu legen. Daher sind stets mindestens sechs Stimmen für die Durchführung des Verfahrens wie auch für die Verbotsentscheidung erforderlich. Ist der nach $\$ 2$ Abs. 2 BVerfGG mit acht Richtern besetzte Senat gerade noch beschlussfähig, weil sechs Richter anwesend sind ( $\$ 15$ Abs. 2 Satz 1 BVerfGG), muss die Entscheidung also einstimmig ergehen. ${ }^{45}$ (2) Für das eigentliche Verbotsverfahren hat das BVerfGG keine näheren Regelungen getroffen. Es richtet sich nach den allgemeinen Verfahrensbestimmungen. Danach muss stets eine mündliche Verhandlung stattfinden ( $\$$ 25 Abs. 1 BVerfGG), sofern nicht alle Beteiligten ausdrücklich darauf verzichten. Die Sachentscheidung ergeht durch Urteil.

Eine Prozessentscheidung ergeht durch Beschluss. So lag der Fall im NPD-Parteiverbotsverfahren, das der Zweite Senat des Bundesverfassungsgerichts am 8. März 2003 durch Beschluss eingestellt hat. Das Verfahren, das Bundesregierung, Bundestag und Bundesrat am 30. Januar beziehungsweise 30. März 2001 beim BVerfG zwecks Feststellung der Verfassungswidrigkeit der NPD und Auflösung ihrer Parteiorganisation eingeleitet hatten, konnte nicht fortgeführt werden, weil der von der Antragsgegnerin NPD sinngemäß gestellte Antrag auf Einstellung des Verfahrens nicht die für eine Ablehnung nach $\$ 15$ Abs. 4 Satz 1 BVerfGG erforderliche Zweidrittelmehrheit gefunden hatte. ${ }^{46}$ An dem Verfahren wirkten sieben Richter mit. Die die Entscheidung tragenden Richter Winfried Hassemer, Siegfried Broß und Lerke Osterloh waren der Auffassung, dass ein nicht behebbares Verfahrenshindernis vorliege. ${ }^{47}$ Die Mehrheit des Senats, die Richter Bertold Sommer, HansJoachim Jentsch, Udo Di Fabio und Rudolf Mellinghoff, meinte, dass ein solches nicht bestehe, und hielt die Fortführung des Verfahrens für geboten. ${ }^{48}$

Die Minderheit sah das nicht behebbare Verfahrenshindernis darin, dass das Gebot strikter Staatsfreiheit der Antragsgegnerin rechtsstaatswidrig verfehlt worden sei. Die drei Richter hielten die Beobachtung einer politischen Partei durch V-Leute staatlicher Behörden,

45 Vgl. Rudolf Mellinghoff, in: Theodor Maunz / Bruno Schmidt-Bleibtreu / Franz Klein / Herbert Bethge, Bundesverfassungsgerichtsgesetz. Kommentar, München 2007, \$15 Rn. 71.

46 Ebenso gilt die Zweidrittelmehrheit nach $\$ 15$ Abs. 4 Satz 1 BVerfGG bei einer nachteiligen Entscheidung in den Verfahren über die Verwirkung von Grundrechten (Art. 18 GG; $₫ 13$ Nr. 1 BVerfGG), über eine Anklage gegen den Bundespräsidenten (Art. 61 GG, $\$ 13$ Nr. 4 BVerfGG) und über Richteranklagen (Art. 98 Abs. 2, 5 GG, $\$ 13$ Nr. 9 BVerfGG).

47 BVerfGE 107, S. 339 (S. 360 ff.).

48 BVerfGE 107, S. 339 (S. 378 ff.). 
die als Mitglieder des Bundesvorstands oder eines Landesvorstands fungieren, unmittelbar vor und während der Durchführung eines Parteiverbotsverfahrens für in der Regel unvereinbar mit den Anforderungen an ein rechtsstaatliches Verfahren.

Die Mehrheit legte für die Annahme eines Verfahrenshindernisses einen strengen Maßstab an. Die nachrichtendienstliche Beobachtung der NPD begründe weder im Hinblick auf den Grundsatz der Staatsfreiheit der Parteien noch wegen Fragen der Zurechnung der vorgelegten Erkenntnismittel noch aufgrund der Pflicht zur Gewährung eines fairen Verfahrens ein Hindernis. Eine staatliche Fremdsteuerung der NPD des Ausmaßes, dass ihr politisches Erscheinungsbild nicht mehr das Ergebnis eines offenen gesellschaftlichen Willensbildungsprozesses sei, sei nicht ansatzweise erkennbar.

Bei einer Einstellung des Verfahrens im Wege einer Prozessentscheidung wegen nicht behebbarer rechtsstaatlicher Verfahrensmängel bleiben erneute Anträge ohne Weiteres möglich. Im Unterschied zu den Rechtsfolgen einer gerichtlichen Sachentscheidung brauchen sie insbesondere nicht auf neue Tatsachen gestützt zu sein $(\$ 47$ in Verbindung mit $\$ 41$ BVerfGG).

\subsection{Verlust der Parteieigenschaft kraft Gesetzes}

Nach $₫ 2$ Abs. 2 PartG verliert eine Vereinigung ihre Rechtsstellung als Partei, wenn sie sechs Jahre lang weder an einer Bundestagswahl noch an einer Landtagswahl teilgenommen hat. Das BVerfG hat diese Vorschrift nicht beanstandet. ${ }^{49}$ Nach seiner Auffassung gehört es zum Parteibegriff des Art. 21 Abs. 1 GG, dass sich eine politische Partei an den Wahlen in Bund und Ländern beteiligt. Daher ist es aus seiner Sicht nur konsequent, dass eine Partei ihren Parteicharakter verliert, wenn sie sich über eine längere Zeit hinaus nicht an solchen Wahlen beteiligt. Wie lang dieser Zeitraum bemessen ist, ergibt sich allerdings nicht unmittelbar aus Art. 21 Abs. 1 GG. Vorausgesetzt wird nur, dass eine Partei ihre Aufgabe, an der Willensbildung des Volkes durch Beteiligung an Wahlen mitzuwirken, innerhalb einer „vernünftigen Zeitspanne“ erfüllen muss. Gemäß Art. 21 Abs. 3 GG ist es Sache des Bundesgesetzgebers, das Nähere zu regeln. Das BVerfG hat dem Bundesgesetzgeber attestiert, die ihm durch Art. 21 Abs. 1 GG gezogenen Grenzen mit $\$ 2$ Abs. 2 PartG nicht überschritten zu haben. Insbesondere schränkt diese Bestimmung die Freiheit der Parteien nicht in verfassungswidriger Weise ein. $\$ 2$ Abs. 2 PartG verlangt lediglich, dass die Parteien zu einer Bundestags- oder Landtagswahl eigene Wahlvorschläge nach den einschlägigen Wahlgesetzen einreichen. Wenn bei einer Bundestagswahl eine politische Vereinigung nur einen Kreiswahlvorschlag einreichen würde, nähme sie an dieser Wahl schon als Partei teil. Sollte eine Partei bisher nicht im Bundestag oder in einem Landtag durch Abgeordnete vertreten gewesen sein, würde sie nur 200 Unterschriften von Wahlberechtigten des Wahlkreises dem Kreisvorschlag beifügen müssen, um als Partei anerkannt zu werden ( $\$ 20$ Abs. 2 BWG). Auch wird durch $\$ 2$ Abs. 2 PartG dem Umstand Rechnung getragen, dass möglicherweise eine Partei aus taktischen Erwägungen zeitweise nicht an Wahlen im Bund oder in den Ländern teilnehmen möchte, zum Beispiel weil sie sich neu formieren oder sich nicht mit der Hypothek eines offenkundigen Misserfolges belasten möchte. 
Die Übereinstimmung der Legaldefinition in $\$ 2$ Abs. 2 Satz 1 PartG mit dem Parteibegriff des Grundgesetzes wird von einem Teil der Literatur entgegen der Auffassung des Bundesverfassungsgerichts für zweifelhaft gehalten. ${ }^{50} \mathrm{Da}$ als prägendes Begriffsmerkmal die Mitwirkung an der Willensbildung des Volkes genannt sei, müssten folgerichtig alle politischen Vereinigungen unter den Parteibegriff fallen, die an der Willensbildung mitwirkten. Die Willensbildung des Volkes vollziehe sich nicht allein bei Bundestags- und Landtagswahlen, sondern in gleicher Weise bei Kommunalwahlen wie auch bei den Wahlen zum Europäischen Parlament. ${ }^{51}$ Mit einer "Sonderrolle“, die namentlich den kommunalen Gebietskörperschaften ${ }^{52}$ und den Kommunalwahlen ${ }^{53}$ in der früheren Rechtsprechung des Bundesverfassungsgerichts zugewiesen wurde, lasse sich diese Einengung des Parteibegriffs heute nicht mehr begründen. ${ }^{54}$

Mit Wegfall des Status als politische Partei verliert die Vereinigung das Parteienprivileg und nimmt nicht mehr an der staatlichen Parteienfinanzierung nach $\$ \$ 18 \mathrm{ff}$. PartG teil. Die rechtliche Infrastruktur als eingetragener beziehungsweise nichtrechtsfähiger Verein bleibt indes erhalten, wenn keine Liquidation erfolgt. Wenn allerdings im Verein über einen „sehr langen Zeitraum hinweg“ keinerlei Tätigkeit zur Verfolgung des Vereinszwecks durch die Mitglieder und die Vereinsorgane ausgeübt worden ist, also der Vereinszweck endgültig aufgegeben worden ist, so wird dieser Fall dem Ausscheiden sämtlicher Mitglieder gleichgestellt. ${ }^{55}$ Nach herrschender Auffassung wird im Falle der endgültigen Aufgabe der Vereinstätigkeit das liquidationslose Erlöschen des Vereins angenommen. ${ }^{56}$

\section{Verschmelzung}

\subsection{Die Gründung der Partei „Die Linke“}

Die Linke ist aus einer Verschmelzung der Parteien Die Linkspartei.PDS und Wahlalternative Arbeit und soziale Gerechtigkeit (WASG) hervorgegangen. Die PDS hat ihre Wurzeln in der 1946 gegründeten Sozialistischen Einheitspartei Deutschlands (SED). Die SED fügte im Dezember 1989 infolge der friedlichen Revolution in der DDR ihrem bisherigen Namen den Anhang PDS hinzu. Im Februar 1990 änderte die SED-PDS ihren Namen in PDS, den sie bis zum 17. Juli 2005 trug, als sie sich in Linkspartei.PDS umbenannte.

Die WASG wurde am 3. Juli 2004 nach dem Zusammenschluss der vor allem in den westdeutschen Ländern aktiven „Initiative Arbeit \& soziale Gerechtigkeit“ (ASG) sowie der Initiative „Wahlalternative“ zunächst als Verein ${ }^{57}$ unter dem Namen „Wahlalternative Ar-

50 Vgl. Jörn Ipsen, in: Michael Sachs, Grundgesetz. Kommentar, München 2007, Art. 21 Rn. 19; Rudolf Streinz, a.a.O. (Fn. 22), Art. 21 Rn. 47.

51 Für eine analoge Anwendung von $\$ 2$ Abs. 2 PartG auf die Teilnahme an Wahlen zum Europäischen Parlament Martin Morlok, in: DVBl. 1989, S. 393.

52 BVerfGE 2, S. 1 (S. 76).

53 BVerfGE 6, S. 367 (S. 372 f.).

54 Vgl. Jörn Ipsen, in: ders., Parteiengesetz, München 2008, $\$ 2$ Rn. 6.

55 Bernhard Reichert, a.a.O. (Fn. 7), Rn. 3733 unter Bezug auf BGHZ 19, S. 51 (S. 61); Bundesgerichtshof, Wertpapiermitteilung 1965, S. 1132 und 1976, S. 686 (S. 687); Bundesarbeitsgericht, in: NJW 1967, S. 1437; BVerwGE 109, S. 273 (S. 281).

56 Vgl. die Zitate ebenda.

57 Wahlalternative Arbeit und soziale Gerechtigkeit e.V. (WAsG e.V.). 
beit und soziale Gerechtigkeit“ gegründet. Am 22. Januar 2005 wurde in Göttingen die Gründung der WASG als Partei unter dem Namen Arbeit \& soziale Gerechtigkeit - Die Wahlalternative beschlossen. Der Gründungsparteitag fand am 7./8. Mai 2005 in Dortmund statt.

Am 7. Juni 2005 einigten sich PDS und WASG grundsätzlich über den Antritt als gemeinsames Linksbündnis bei der vorgezogenen Bundestagswahl 2005. Da eine Listenverbindung ohne Zusammenschluss von WASG und PDS im bundesdeutschen Wahlrecht nicht möglich war ${ }^{58}$, verzichtete die WASG zugunsten der PDS auf eine eigene Teilnahme an der Bundestagswahl. Stattdessen kandidierten Vertreter der WASG auf offenen Listen der PDS ${ }^{59}$, die sich in Die Linkspartei.PDS (Kurzform „Die Linke“) umbenannte, wobei es den Landesverbänden freistand, den Namensbestandteil PDS zu verwenden. Die Linkspartei.PDS erhielt bei der Bundestagswahl 2005 8,7 Prozent der Zweitstimmen. Der Erfolg des linken Wahlbündnisses und die Bildung einer gemeinsamen Fraktion im Herbst 2005 förderten den weiteren Fusionsprozess. Die Fusion sollte durch Parteitagsbeschlüsse und Urabstimmungen bis spätestens 30. Dezember 2007 durchgeführt werden. Am 10. Dezember 2006 beschlossen die Vorstände beider Parteien in einer gemeinsamen Sitzung die gemeinschaftlich erarbeiteten Entwürfe der Gründungsdokumente für die Partei Die Linke (unter anderem Namen, Programmatik, Satzung, Finanz- und Schiedsordnung sowie Rahmenwahlordnung) als Vorlage für die parallel tagenden Bundesparteitage beider Parteien in Dortmund am 24./25. März 2007. ${ }^{60}$ Auf diesen Parteitagen stimmten die Delegierten beider Parteien mit großer Mehrheit den gleichlautenden Gründungsdokumenten sowie dem Verschmelzungsvertrag zu. Danach führten beide Parteien vom 30. März bis 18. Mai 2007 Urabstimmungen unter ihren Mitgliedern über die Parteitagsbeschlüsse durch. Bei der Linkspartei stimmten 96,9 Prozent und bei der WASG 93,9 Prozent für die Fusion. ${ }^{61}$ Der Gründungsparteitag des verschmolzenen Vereins „Die Linkspartei e.V.“ fand am 16. Juni 2007 in Berlin statt. ${ }^{62}$ Im Vereinsregister des Amtsgerichts Charlottenburg findet sich unter „Sonstige Rechtsverhältnisse“ als Registerinhalt: „Mit dem Verein (übernehmender Rechtsträger) ist aufgrund des Verschmelzungsvertrages vom 24. Mai 2007 sowie der Beschlüsse der Delegiertenversammlungen vom 25. März 2007 des Arbeit und soziale Gerechtigkeit - Die Wahlalternative e.V. mit Sitz in Berlin (Amtsgericht Charlottenburg, VR 26146 B) durch Übertragung des Vermögens als Ganzes verschmolzen."

58 Vgl. dazu Jürgen Schilde, Zur Frage mehrparteiiger Listenverbindungen und Kooperation bei der Bundestagswahl, Ausarbeitung WF III - 306/04 der Wissenschaftlichen Dienste des Deutschen Bundestages (nicht veröffentlicht).

59 Durch Art. 1 des Gesetzes vom 17. März 2008 (BGBl. I, S. 394) wurde $\$ 21$ Abs. 1 Satz 1 des Bundeswahlgesetzes geändert und erhielt folgenden Wortlaut: „Als Bewerber einer Partei kann in einem Kreiswahlvorschlag nur benannt werden, wer nicht Mitglied einer anderen Partei ist und in einer Mitgliederversammlung zur Wahl eines Wahlkreisbewerbers oder in einer besonderen oder allgemeinen Vertreterversammlung hierzu gewählt worden ist."

60 Siehe dazu auch den Bericht zur Verschmelzung der Parteien „Die Linkspartei.PDS“ und „Arbeit \& soziale Gerechtigkeit - Die Wahlalternative“: http://www.die-Linke.de/index.php?id=181\&tx_ ttnews[tt_news]=1090\&tx_ttnews[backPid]=154\&no_cache=1 (Abruf am 11. Februar 2009).

61 Vgl. zum Ganzen auch Kolja Bartsch, Die Vereinigung von Linkpartei.PDS und WASG zur Partei Die Linke, in: Aktueller Begriff, Nr. 38 vom 12. Juni 2007, Wissenschaftliche Dienste des Deutschen Bundestages.

62 Siehe dazu die Internetseite der Partei Die Linke: http://die-linke.de/partei/organe/parteitage/ gruendungsparteitag/ (Abruf am 11. Februar 2009). 


\subsection{Differenz zwischen verfassungsrechtlicher und einfachgesetzlicher Ebene}

Die Fusion von zwei Parteien lässt sich als Sonderfall der Parteigründung verstehen und genießt damit den verfassungsrechtlichen Schutz des Art. 21 Abs. 1 GG. ${ }^{63}$ Nach Satz 2 dieses Parteiengrundrechts ist die Gründung der Parteien frei.

Die Gründungsfreiheit beinhaltet auch die Freiheit der Wahl der Rechtsform. Da die Parteien im Raum der Gesellschaft angesiedelte Gebilde darstellen, kommen dabei nur die Rechtsformen des Privatrechts und hier nach den gesetzlichen Vorgaben nur diejenigen des rechtsfähigen oder nichtrechtsfähigen Vereins in Betracht (\$\$21, 54 BGB). ${ }^{64}$ Die Vorschriften des privaten Vereinsrechts gelten auch für Parteien, soweit sie nicht ausdrücklich davon ausgenommen sind (vgl. \$ 37 PartG). Die Gründungsfreiheit der Parteien aus Art. 21 Abs. 1 Satz 2 GG und ihre Betätigungsfreiheit implizieren auch die Organisationsfreiheit. Als Recht der Partei bedeutet dies, dass sie sich durch internen Mehrheitsbeschluss auf bestimmte organisatorische Gestaltungen festlegen und diese dann auch ohne staatliche Behinderung realisieren darf. ${ }^{65}$ Welche Gesichtspunkte den Organisationsentscheidungen einer Partei zugrunde liegen, ist für das staatliche Recht unbeachtlich. Insbesondere dürfen auch Überlegungen politischer Zweckmäßigkeit für Organisationsentscheidungen ausschlaggebend sein. Raison d'être von Parteien ist es, Mehrheiten zu gewinnen und möglichst große Anteile an Wählerstimmen zu erlangen. Es gehört von daher zur inhärenten Logik einer Parteiendemokratie, dass die Parteien versuchen, organisatorische Voraussetzungen für einen möglichst großen Wahlerfolg zu schaffen. ${ }^{66}$

Der Verfassungsbegriff der politischen Parteien nach Art. 21 GG kann nicht durch einfaches Bundesgesetz bestimmt werden. ${ }^{67}$ Bei $\$ 2$ Abs. 1 PartG handelt es sich deshalb nicht um eine authentische Interpretation. ${ }^{68}$ Die Verfassungsinterpretation kann sich zwar an der Parteiendefinition von $\$ 2$ Abs. 1 PartG orientieren, ihr kommt jedoch nur Hilfsfunktion zu. Der Parteibegriff ist durch Art. 21 Abs. 1 Satz 1 GG vorgegeben und eröffnet keinen Gestaltungsspielraum für den Gesetzgeber. Eine Definitionskompetenz des Gesetzgebers könnte zu Einengungen des Parteibegriffs führen, die die Sicherungen des Art. 21 GG wirkungslos werden ließen. ${ }^{69}$ Ungeachtet dessen hat das BVerfG den Parteibegriff des $₫ 2$ Abs. 1 PartG für verfassungsmäßig erklärt. ${ }^{70}$

63 Vgl. Martin Morlok, Gutachten zu Fragen einer Fusion zwischen WASG und Linkspartei, http://www.axel-roost.de/article/447.morlok_gutachten_zur_fusion_von_wasg_und_linkspartei. htm (Abruf am 11. Februar 2009); ders., in: Horst Dreier, a.a.O. (Fn. 33), Art. 21 Rn. 58; Gerd Roellecke, in: Peter C. Umbach / Thomas Clemens, Grundgesetz. Mitarbeiterkommentar und Handbuch, Heidelberg 2002, Art. 21 Rn. 66; Uwe Volkmann, in: Karl Heinrich Friauf/ Wolfram Höfling, Berliner Kommentar zum Grundgesetz, Berlin 2001, Art. 21 Rdnr. 40.

64 Statt aller Hans Hugo Klein, a.a.O. (Fn. 22), Art. 21 Rn. 274.

65 Vgl. Martin Morlok, a.a.O. (Fn. 63), S. 8.

66 Vgl. ebenda.

67 Vgl. ders., in: Horst Dreier, a.a.O. (Fn. 33), Art. 21 Rn. 33.

68 Vgl. Rudolf Streinz, a.a.O. (Fn. 22), Art. 21 Rn. 46; Philip Kunig, a.a.O. (Fn. 29), Art. 21 Rn. 12.

69 Vgl. Jörn Ipsen, a.a.O. (Fn. 54), $\$ 2$ Rn. 2.

70 Zum Beispiel BVerfGE 47, S. 198 (S. 222); BVerfGE 91, S. 276 (S. 284); BVerfGE 98, S. 266 (S. 269 ff.). 
Nach alldem besteht also eine Ebenendifferenz zwischen verfassungsrechtlichem und einfachgesetzlichem Parteibegriff, die in einem konkreten Anwendungsfall des einfachen Gesetzes funktional aufzulösen ist. Dies hat besondere Bedeutung bei der Parteienfusion.

\$\$ 6 Abs. 2 Nr. 11 und 9 Abs. 3 PartG erwähnen die Verschmelzung politischer Parteien nur insoweit, als sie die Zuständigkeit des Parteitages für die Beschlussfassung über die Verschmelzung regeln, ohne eine materielle Rechtsgrundlage für die Fusion von Parteien zu bilden. ${ }^{71}$ Die Verschmelzung von Parteien richtet sich nach den allgemeinen Vorschriften des Zivilrechts, da die Parteien in der Rechtsform des eingetragenen oder nichtrechtsfähigen Vereins organisiert sind.

Vor Inkrafttreten des Umwandlungsgesetzes (UmwG) vom 28. Oktober $1994^{72}$ war die Umwandlung von Vereinen nur in sehr engem Rahmen möglich. Einheitliche Regelungen fehlten gänzlich. Nur in Einzelfällen ließ das Gesetz die Beteiligungen von Vereinen an Umwandlungen zu. Eines der Ziele des UmwG von 1994 war es, die Umstrukturierungsmöglichkeiten von Verbänden, die zuvor in fünf verschiedenen Gesetzen ${ }^{73}$ geregelt waren, zu vereinheitlichen und zusammenzufassen. Gleichzeitig sollten Lücken der bisherigen Regelungen geschlossen werden. Hierzu gehörte auch die Einführung der Beteiligung von Vereinen an Umwandlungen. Das UmwG ermöglicht es nunmehr auch rechtsfähigen Vereinen, sich in erleichterter Form umzuwandeln, zum Beispiel zu verschmelzen $(\$ \$ 2$ bis $122 \mathrm{UmwG}$ ). Bei der Verschmelzung findet eine rechtsgeschäftliche, unter anderem im Verschmelzungsvertrag geregelte, vollständige oder teilweise Gesamtrechtsnachfolge statt, was sich vor allem auf das Vermögen der beteiligten Rechtsträger auswirkt. Zwei Arten der Verschmelzung sind zu unterscheiden ( $\$ 2$ UmwG): die Verschmelzung durch Aufnahme und die Verschmelzung durch Neugründung.

Bei der Verschmelzung durch Aufnahme ( $\$ 2$ Nr. 1 UmwG) übertragen ein oder mehrere Rechtsträger unter Auflösung ohne Abwicklung ihr Vermögen als Ganzes auf einen anderen, bereits bestehenden Rechtsträger. Die Wirkungen der Verschmelzung durch Aufnahme treten - ohne dass es eines weiteren Rechtsaktes bedarf - mit deren Eintragung im Vereinsregister des übernehmenden Vereins ein ( $\$ 20$ Abs. 1 UmwG). Die Wirkungen dieser rechtsgeschäftlichen Gesamtrechtsnachfolge beziehen sich auf das Vermögen, auf Verbindlichkeiten, Beteiligungen sowie Forderungen und Schulden. Die Verschmelzung hat weiter zur Folge, dass (nur) der übertragende Verein erlischt ( $\$ 20$ Abs. 1 Nr. 2 Satz 1 UmwG). Die vorher nicht ausgetretenen Mitglieder des übertragenden Vereins werden Mitglieder des übernehmenden Vereins (\$20 Abs. 1 Nr. 3 Halbsatz 1 UmwG). Das Mitgliedschaftsverhältnis im übertragenden Verein setzt sich im Wege des Anteilsaustausches ${ }^{74} \mathrm{im}_{\text {aufneh- }}$ menden Verein fort.

71 Vgl. Ralph Katschinski, in: Johannes Semmler / Arndt Stengel (Hrsg.), Umwandlungsgesetz, München 2007, $₫ 99$ Rn. 3; Karl-Heinz Seifert, a.a.O. (Fn. 6), S. 174; anderer Ansicht Ulrich Drobnig / Michael Becker / Oliver Remien, Verschmelzung und Koordinierung von Verbänden, Tübingen 1991, S. 25, die davon ausgehen, dass die $\$ \$ 6$ Abs. 2 Nr. 11 und 9 Abs. 3 PartG nicht nur Zuständigkeitsregelungen darstellen, sondern auch materiellrechtlich die Verschmelzung von Parteien ermöglichen.

72 BGBl. I, S. 3210, zuletzt geändert durch Art. 1 des Gesetzes vom 19. April 2007 - BGBl. I, S. 542.

73 UmwG 1969; $\$ \$ 339$ ff. AktG alte Fassung; $\$ \$ 19$ ff. KapErhG; $\$ \$ 63$ cff., 93a ff. GenG; $\$ \$ 44 a$ ff., 53 a VAG.

74 Vgl. Bernhard Reichert, a.a.O. (Fn. 7), Rn. 4127. 
Bei der Verschmelzung von Vereinen durch Neubildung ist der übernehmende Verein nicht ein bereits bestehender, sondern ein von den an der Verschmelzung beteiligten Vereinen neu gegründeter Verein. Mit dieser Abweichung findet die Verschmelzung durch Neubildung wie eine Verschmelzung durch Aufnahme in einen bestehenden Verein statt. Entweder im Verschmelzungsvertrag oder gesondert muss die Satzung für den neuen Verein errichtet werden. ${ }^{75}$

Nach der Rechtsprechung des Bundesverfassungsgerichts statuiert Art. 21 Abs. 1 Satz 2 GG für politische Parteien die Gründungs- und Betätigungsfreiheit, die sich auch auf die Organisations- und Programmfreiheit erstreckt. Eine Partei ist frei in der Wahl ihrer identitätsbestimmenden Merkmale, in der Gestaltung ihrer politischen Ziele, in der Ausrichtung ihrer Programmatik und in der Wahl ihrer Themen. Erhebt sie gesellschaftliche Themen, die ausschließlich in einem Land wurzeln, zum Programm und beschränkt sich in der Auseinandersetzung auf die Einflussnahme auf die politische Willensbildung der Bevölkerung eines Landes mit dem Ziel, sich in deren Volksvertretung mit „ihren“ Themen Geltung zu verschaffen, so ist dies nach Art. 21 Abs. 1 GG in gleicher Weise „förderungswürdig und schützenswert" wie die politische Tätigkeit einer Partei, die Vorgänge mit länderüberschreitendem Interesse aufgreift und im politischen Wettbewerb bundesweit thematisiert. ${ }^{76}$

Diesen Ausführungen des Bundesverfassungsgerichts ist eine Förderungs- und Schutzpflicht des Staates bei der Gründung der Partei zu entnehmen. Der Staat hat alles zu unterlassen, was die Gründung der Partei verhindern oder behindern würde. Aus der Gründungsfreiheit folgt das Recht auf das „Entstehen“ einer Partei. ${ }^{77}$ Dem ist Genüge getan, wenn der Staat ein rechtstechnisches Regelwerk zur Verfügung stellt, das den Parteien die Gründung ermöglicht. Dies gilt insbesondere für die vermögensrechtliche „Startsituation“. Es ist die Konstruktion zu wählen, die der gegründeten Partei die beste materielle Ausgangsposition verschafft. Dies bedeutet für den Fall der Verschmelzung von Parteien, dass sie ihr erworbenes Vermögen in die neu entstehende Partei einbringen dürfen mit allen Anwartschaften auf die staatliche Parteienfinanzierung, denn die fusionierenden Parteien können nach der Verschmelzung nicht schlechter gestellt sein als vorher. Deshalb ist es unter der verfassungsrechtlichen Ägide des Art. 21 GG unerheblich, ob die Verschmelzung rechtstechnisch im Sinne des Umwandlungsgesetzes eine Verschmelzung durch Aufnahme oder durch Neugründung darstellt. Im Falle der Partei Die Linke ist die aufnehmende Partei die Linkspartei.PDS gewesen, so dass rein zivilrechtlich die neue Partei Die Linke mit der PDS rechtsidentisch ist. Dennoch ist eine neue Partei entstanden, nämlich nach ihrem erklärten Willen, der verfassungsrechtlich nicht ignoriert werden kann. Diese verfassungsrechtliche Situation und die zivilrechtliche Seite sind, was die Frage der Rechtsidentität angeht, dualistisch und nicht monistisch zu betrachten. Eine Ebenendifferenz zwischen Verfassungs- und Zivilrecht ist im Übrigen nichts Ungewöhnliches, denkt man etwa an die Eigentumsgarantie des Art. 14 GG. Bereits für Art. 153 WRV war allgemein die Anschauung anerkannt, dass das Eigentumsgrundrecht nicht nur das sachenrechtliche Eigentum, sondern jedwedes „private Vermögensrecht“ gewährleistet. Unter der Geltung des Grundgesetzes hat sich daran nichts geändert. ${ }^{78}$

75 Vgl. ebenda, Rn. 4233 ff.

76 BVerfGE 111, S. 382 (S. 409).

77 Philip Kunig, a.a.O. (Fn. 29), Art. 21 Rn. 50.

78 Rudolf Wendt, in: Michael Sachs, a.a.O. (Fn. 50), Art. 14 Rn. 22. 